\title{
Quality of Mental Health Care in Integrated Veterans Affairs Patient-Centered Medical Homes: a National Observational Study
}

\author{
Lucinda B. Leung, MD, PhD, MPH ${ }^{1,2}$, Edward P. Post, MD, PhD ${ }^{3,4}$, Erin Jaske, MPH ${ }^{5}$, \\ Kenneth B. Wells, MD, MPH ${ }^{1,6}$, and Lisa V. Rubenstein, MD, MSPH $H^{2,7}$
}

\begin{abstract}
'Center for the Study of Healthcare Innovation, Implementation, \& Policy, VA Greater Los Angeles Healthcare System, Los Angeles, CA, USA; ${ }^{2}$ Division of General Internal Medicine and Health Services Research, VA Greater Los Angeles Healthcare System, UCLA David Geffen School of Medicine, Los Angeles, CA, USA; ${ }^{3}$ Center for Clinical Management Research, VA Ann Arbor, Ann Arbor, MI, USA; ${ }^{4}$ Department of Medicine, University of Michigan Medical School, Ann Arbor, MI, USA; ${ }^{5}$ VHA Primary Care Analytics Team, VA Puget Sound Health Care System, Seattle, WA, USA; ${ }^{6}$ Department of Psychiatry and Biobehavioral Sciences, UCLA Semel Institute for Neuroscience and Human Behavior, Los Angeles, CA, USA; ${ }^{7}$ RAND Corporation, Santa Monica, CA, USA.
\end{abstract}

J Gen Intern Med 34(12):2700-1 DOI: $10.1007 / \mathrm{s} 11606-019-05310-1$

(C) Society of General Internal Medicine (This is a U.S. government work and not under copyright protection in the U.S.; foreign copyright protection may apply) 2019

\section{INTRODUCTION}

Mental health integration in primary care is effective but challenging to disseminate and implement in health care systems. ${ }^{1}$ Over the past decade, the Veterans Health Administration (VA) transformed primary care practices nationally into team-based care models: Primary Care-Mental Health Integration (PC-MHI) ${ }^{2}$ (i.e., evidence-based collaborative care) in 2007 and Patient Aligned Care Teams (PACT) ${ }^{3}$ (i.e., patientcentered medical home) in 2010. Both initiatives provide staffing and resources to support primary care providers, care managers, and integrated mental health specialists in jointly treating low-to-moderate severity psychiatric conditions within primary care. ${ }^{2}$ Primary care has been tasked with universal mental health screening and expected to initiate timely treatment, aided by PC-MHI, for Veterans identified with needs. As such, mental health care has accordingly shifted from VA specialty to PACT/primary care settings. ${ }^{4}$ This study examined whether increasing penetration of PC-MHI services among PACT clinics was associated with improved mental health care process quality measures for VA primary care patients.

\section{METHODS}

In an ongoing VA quality improvement (non-research) effort, we retrospectively observed 828,050 Veterans receiving primary care from VA clinics mandated to offer PC-MHI services

Prior Presentations A previous version of this article was presented at the AcademyHealth Annual Research Meeting in Washington, D.C. on June 2, 2019.

Published online September 4, 2019 from October 1, 2013, to September 30, 2016 ( $n=396$ clinics nationally). As a proxy for care integration in each clinic, we calculated clinic PC-MHI penetration, which was the proportion of assigned primary care patients seen by a PC-MHI provider ${ }^{\text {annually } 4}$ (median $=6.7 \%$; interquartile range $=4.7-$ $8.7 \%$ ). Given that PACT is universally required in the VA, we calculated each clinic's PACT implementation Progress Index $\left(\mathrm{PI}^{2}\right)$, which measures the extent of medical home implementation using patient/provider surveys and administrative data and was newly available preceding our study. ${ }^{5}$ Trained External Peer Review Program abstractors reviewed patient electronic health records for Healthcare Effectiveness Data and Information Set (HEDIS) analogous clinical quality measures for psychiatric illness screening and management (Table 1). We reported the average proportion of patients meeting each measure in each clinic. In multilevel logistic regressions, we used clinic PC-MHI penetration to predict odds of timely follow-up for newly abnormal disease screening. Models controlled for year, $\mathrm{PI}^{2}$, regional health care system, clinic characteristics, patient characteristics, and an additional interactive effect between clinic PC-MHI penetration and $\mathrm{PI}^{2}$ in sensitivity analyses.

\section{RESULTS}

Clinic screening rates for depression, posttraumatic stress disorder (PTSD), and alcohol misuse were high (on average, 96\%, 99\%, and 97\%). Averages rates of clinician follow-up within $24 \mathrm{~h}$ following newly abnormal screening were $64 \%$ (depression), 79\% (PTSD), and 79\% (alcohol misuse). In fully adjusted models, there were no PC-MHI, $\mathrm{PI}^{2}$, or interactive effects observed among all mental health care quality indicators (Table 1). We did not observe any significant differences in care quality due to differences in gender, racial-ethnicity, homelessness, physical comorbidity, and serious mental illness (schizophrenia, bipolar disorder). There were few differences in quality measures associated with patient age. With 
Table 1 Effect of Clinic PC-MHI Penetration on Mental Health Care Quality, 2013-2016

\begin{tabular}{|c|c|c|c|c|c|}
\hline Quality measure completed within $24 \mathrm{~h}$ & Clinic mean & Odds ratio & 95\% CI, lower limit & 95\% CI, upper limit & $\overline{p \text { value }}$ \\
\hline \multicolumn{6}{|l|}{ Suicide risk evaluation } \\
\hline Following annual positive depression screen & $83 \%$ & 0.99 & 0.92 & 1.07 & 0.80 \\
\hline Following annual positive PTSD screen & $84 \%$ & 0.96 & 0.88 & 1.04 & 0.33 \\
\hline Following annual positive depression or PTSD & $83 \%$ & 0.98 & 0.93 & 1.04 & 0.54 \\
\hline \multicolumn{6}{|l|}{ Clinician follow-up or counseling } \\
\hline Following annual positive depression screen & $64 \%$ & 0.96 & 0.90 & 1.03 & 0.29 \\
\hline Following annual positive PTSD screen & $79 \%$ & 0.95 & 0.85 & 1.06 & 0.36 \\
\hline $\begin{array}{l}\text { For alcohol misuse screening result of } 5 \text { or greater } \\
\text { (within } 14 \text { days) }\end{array}$ & $79 \%$ & 0.97 & 0.92 & 1.02 & 0.20 \\
\hline
\end{tabular}

PC-MHI Primary Care-Mental Health Integration, CI confidence interval, PTSD post-traumatic stress disorder

Key quality metrics examined and thought to be facilitated by primary care and mental health integration are shown. Multilevel logistic regression models controlled for clinic PC-MHI penetration rate, year, VA regional network, clinic characteristics (level of patient-centered medical home implementation, size, rurality, type [i.e., hospital, community-based]), and patient characteristics (age, gender, race-ethnicity, marital status, VA means test, service connectedness, Gagne Comorbidity Score category, homelessness, distance from home to clinic, depression, anxiety, PTSD, substance use disorder, serious mental illness [i.e., schizophrenia, bipolar disorder]). The distribution of clinic PC-MHI penetration was normalized by performing log base 2 transformation. Odds ratios, therefore, are interpreted as percentage changes in odds of a person meeting the quality measure per year, relative to each two-fold increase in clinic PC-MHI penetration (i.e., a change from $2 \%$ to $4 \%$ of primary care patients seen by a PC-MHI provider annually).

each increasing year of patient age, odds were $1 \%$ higher in timely clinician follow-up for depression $(p=0.04)$ and suicide risk evaluation for PTSD $(p=0.01)$.

\section{DISCUSSION}

As PC-MHI services were increasingly used in VA primary care, mental health care quality (i.e., timely follow-up of abnormal disease screening) was high. PC-MHI was not associated with care quality, but has engaged more patients in care and reduced their reliance on specialty-based mental health resources. ${ }^{4}$ Variable fidelity to evidence-based collaborative care models as nationally implemented and/or difficulty measuring integration with administrative data may contribute to findings. Nonetheless, we highlight opportunities for mental health care quality improvement in primary care (i.e., less timely clinician follow-up for abnormal depression screening, compared with PTSD, respectively $64 \%$ and $79 \%$ ). Contrary to findings from other large health care systems, ${ }^{6}$ we observed no differences in mental health care quality for racial-ethnic minority patients, nor for vulnerable patients (i.e., homeless, serious mental illness), perhaps because the VA is an experienced safety-net provider. Our correlational study, however, does not permit causal inference. We did not examine patient outcomes and cannot exclude coding inaccuracies within administrative data. Additional research, which better controls for socioeconomic factors, is needed to confirm the lack of racial-ethnic differences observed among VA mental health care performance metrics. Nonetheless, lessons can be learned from VA's national dissemination of two complex team-based care models: PC-MHI and PACT. Results highlight remaining mental health quality gaps and encourage continued optimization of collaborative care models to improve mental health care processes in patient-centered medical homes.

Acknowledgments: Our thanks go to the PACT Mental Health workgroup members (Ranak Trivedi, PhD, Leslie Taylor, PhD, among others) who contributed invaluable expertise and feedback. Quality measures were obtained from the VA External Peer Review Program, which is administered by the VA Office of Reporting, Analytics, Performance, Improvement \& Deployment.

Corresponding Author: Lucinda B. Leung, $M D, P h D, M P H$; Division of General Internal Medicine and Health Services ResearchVA Greater Los Angeles Healthcare System, UCLA David Geffen School of Medicine, Los Angeles, CA, USA (e-mail: lleung@mednet.ucla.edu).

Funding Information This work was undertaken as part of the Veterans Administration's Primary Care Analytics Team, supporting and evaluating VA's transition to a patient-centered medical home. Funding for the Primary Care Analytics Team is provided by the VA Office of Primary Care.

\section{Compliance with Ethical Standards:}

Conflict of Interest: The authors declare that they do not have a conflict of interest.

Disclaimer: The views expressed in this article are those of the authors and do not necessarily reflect the position or policy of the Department of Veterans Affairs or the US government.

\section{REFERENCES}

1. Katzelnick DJ, Williams MD. Large-Scale Dissemination of Collaborative Care and Implications for Psychiatry. Psychiatr Serv. 2015;66(9):904-906.

2. Post EP, Metzger M, Dumas P, Lehmann L. Integrating mental health into primary care within the Veterans Health Administration. Fam Syst Health. 2010;28(2):83-90.

3. Rosland AM, Nelson $\mathbf{K}$, Sun $\mathbf{H}$, et al. The patient-centered medical home in the Veterans Health Administration. Am J Manag Care. 2013; 19(7):e263-272.

4. Leung LB, Rubenstein LV, Yoon J, et al. Veterans Health Administration Investments In Primary Care And Mental Health Integration Improved Care Access. Health Affairs. 2019;38(8):1281-8.

5. Nelson KM, Helfrich C, Sun $\mathbf{H}$, et al. Implementation of the patientcentered medical home in the Veterans Health Administration: associations with patient satisfaction, quality of care, staff burnout, and hospital and emergency department use. JAMA Intern Med. 2014;174(8):13501358.

6. Waitzfelder B, Stewart C, Coleman KJ, et al. Treatment Initiation for New Episodes of Depression in Primary Care Settings. J Gen Intern Med. 2018;33(8):1283-1291.

Publisher's Note Springer Nature remains neutral with regard to jurisdictional claims in published maps and institutional affiliations. 